\title{
Relationship between Suicidality and Low Self-esteem in Patients with Schizophrenia
}

\author{
Taeyoung Yoo', Sung-Wan Kim²,2, Seon-Young Kim¹, Ju-Yeon Lee ${ }^{1,2}$, Hee-Ju Kang', Kyung-Yeol Bae', \\ Jae-Min Kim ${ }^{1}$, II-Seon Shin', Jin-Sang Yoon ${ }^{1}$ \\ ${ }^{1}$ Department of Psychiatry, Chonnam National University Medical School, ${ }^{2}$ Gwangju Bukgu Community Mental Health Center, Gwangju, Korea
}

\begin{abstract}
Objective: Low self-esteem is associated with suicide risk in the general psychiatric population. The aim of this study was to examine associations between suicidality and self-esteem in patients with schizophrenia.

Methods: Subjects meeting the Diagnostic and Statistical Manual of Mental Disorders, 4th edition (DSM-IV) diagnostic criteria for schizophrenia were enrolled. Sociodemographic and clinical variables, including previous suicide attempt history, were assessed. Psychopathology, self-esteem, and self-perceived stigma were also measured using the Positive and Negative Syndrome Scale, the Rosenberg Self-Esteem Scale (SES), the Beck Depression Inventory (BDI), the Beck Hopelessness Scale, and the Korean version of the Internalized Stigma of Mental IIIness scale (K-ISMI).

Results: Of the total of 87 participants, 20 (23.0\%) had attempted suicide. Patients with a history of suicide attempts had significantly higher scores on the $\mathrm{BDI}(p=0.036)$ and $\mathrm{K}-\mathrm{ISMI}(p=0.009)$, and significantly lower scores on the SES ( $p=0.001)$. Analysis of covariance revealed that the SES scores were significantly lower in patients with a history of previous suicide attempts than in those with no history, after controlling for K-ISMI and BDI scores $(p=0.039)$.

Conclusion: Low self-esteem appears to represent a psychological dimension that is closely related to suicide risk. Therefore, clinical attention should be paid to the evaluation and enhancement of low self-esteem in schizophrenia patients with suicidality. A longitudinal prospective study is required to ascertain whether low self-esteem leads suicide attempts.
\end{abstract}

KEY WORDS: Schizophrenia; Suicide; Self-esteem; Stigma; Depression.

\section{INTRODUCTION}

Suicide is a major cause of death in patients with schizophrenia. The risk of suicide in patients with schizophrenia is consistently reported as 20-50 fold higher than that in the general population. Patients with schizophrenia also exhibit early signs of more serious suicidal behavior, ${ }^{1-7)}$ their lifetime suicide rate has been reported as $10 \%$, with more recent studies indicating a rate of $4-5 \% .^{8-11)}$ Moreover, approximately $50 \%$ of patients with schizophrenia attempt suicide during their lifetime. ${ }^{12)}$ This illustrates that suicide accounts for a large proportion of total deaths in patients with schizophrenia. Therefore, identifying individuals at risk of suicide, and making efforts toward its prevention, is particularly important in patients

\footnotetext{
Received: June 5, 2015 / Revised: July 30, 2015

Accepted: July 31, 2015

Address for correspondence: Sung-Wan Kim, MD, PhD Department of Psychiatry, Chonnam National University Medical School, 160 Baekseo-ro, Dong-gu, Gwangju 61469, Korea Tel: +82-62-220-6148, Fax: +82-62-225-2351 E-mail:swkim@chonnam.ac.kr
}

with schizophrenia.

Various risk factors for suicide in patients with schizophrenia have been reported, including previous history of suicide attempts, comorbid depressive disorders, comorbid alcohol and drug misuse, agitation or motor restlessness, fear of mental disintegration, poor adherence to treatment and a recent loss event, younger age, male gender, higher level of education, presence of insight, active hallucinations and delusions, comorbid chronic physical illness and a family history of suicide. ${ }^{13,14)}$ Our previous study of Korean schizophrenia inpatients demonstrated that previous history of suicide attempts, depression as mentioned and a later age of illness onset, poor familial support, and family history of completed suicide, depression, and substance abuse were associated with greater suicide risk. ${ }^{4)}$

Several studies have shown that low self-esteem is related to increased suicide risk. ${ }^{7,13,15,16)}$ The majority of these studies included patients with depressive disorders; few studies have investigated the association between self-esteem and suicide risk in patients with schizo-

() This is an Open-Access article distributed under the terms of the Creative Commons Attribution Non-Commercial License (http://creativecommons.org/licenses/by-nc/4.0) which permits unrestricted non-commercial use, distribution, and reproduction in any medium, provided the original work is properly cited. 
phrenia, although in one previous study self-esteem was associated with quality of life and suicidal ideation in this population. ${ }^{17)}$

Although several previous studies have suggested that low self-esteem may be associated with suicide risk in the general psychiatric population, evidence of these relationships in patients with schizophrenia is lacking. Therefore, in the present study, we examined the associations between self-esteem and suicidality (defined as a previous history of suicide attempts) in patients with schizophrenia.

\section{METHODS}

\section{Subjects}

All subjects were patients recruited either from the psychiatry clinic of Chonnam National University Hospital, the Department of Psychiatry in Naju National Mental Hospital or the Gwangju Bukgu Community Mental Health Center. The psychiatrists evaluated and diagnosed the patients using the Diagnostic and Statistical Manual of Mental Disorders, 4th edition (DSM-IV); subjects meeting DSM-IV ${ }^{18)}$ diagnostic criteria for schizophrenia were enrolled. Recruitment was conducted between October, 2013 and December, 2014.

The key inclusion criteria were: (i) psychiatric patient $\geq 17$ years of age; (ii) DSM-IV diagnosis of schizophrenia; (iii) Korean as a first language; and (iv) ability to complete the questionnaire and provide informed consent. The exclusion criteria were: (i) significantly clinically unstable and uncontrolled medical disease; and (ii) inability to attend psychiatric evaluations and maintain treatment due to another significant physical problem requiring immediate intervention and treatment.

The study was approved by the institutional review boards of all relevant hospitals. All subjects provided written informed consent prior to their participation.

\section{Sociodemographic and Clinical Characteristics}

Sociodemographic characteristics were assessed by a clinical coordinator using a structured case report form. Data on age, gender, education level, and duration of illness were collected.

The clinical characteristics of patients, including type of antipsychotic medications, use of antidepressants or mood stabilizers (lithium, lamotrigine, or valproic acid), alcohol use, and smoking history were collected.

Several assessment scales were used to assess various symptoms and functional aspects; the scales were administered by a psychiatrist and clinical research coordi- nator. Psychopathology was measured using the Positive and Negative Syndrome Scale (PANSS) ${ }^{19,20)}$ administered by the psychiatrist to evaluate psychotic symptoms, the Beck Depression Inventory (BDI), ${ }^{21,22)}$ which assesses subjective depressive symptoms, and the Beck Hopelessness Scale (BHS), ${ }^{23)}$ which assess feelings of hopelessness these latter two instruments were completed by the patient. The Rosenberg Self-Esteem Scale (SES) was also applied; this instrument has been used to assess self-esteem in numerous populations and includes items scored using a 4-point Likert-type scale, ranging from strongly disagree to strongly agree. ${ }^{24)}$ The SES has demonstrated excellent internal consistency. Self-perceived stigma was measured using the Korean version of the Internalized Stigma of Mental Illness scale (K-ISMI), ${ }^{25)}$ which assesses the subjective experience of stigma using sub-scales measuring alienation, stereotype endorsement, perceived discrimination, stigma resistance, and social withdrawal. This instrument is also self-completed; higher scores indicate higher levels of internalized stigma.

\section{Previous Suicidal Attempt}

Lifetime history of suicide attempts was attained using a semi-structured clinical interview. A 'suicide attempt' was defined as a self-reported self-harming behavior that occurred at any time prior to the baseline assessment, and was accompanied by at least some intent to die regardless of the objective lethality of the action. Ambivalent intent to die at the time of a deliberate self-harm act was included in the definition of a suicide attempt. However, self-injurious behaviors with no suicidal intent, or unknown intent, were excluded from the definition in this analysis. The primary group comparison was made between patients reporting at least one previous suicide attempt (referred to as "a history of a previous suicide attempts" for brevity) and those reporting no previous attempts.

\section{Statistical Analysis}

Subjects were divided into two groups according to whether they had previously made a suicide attempt. Sociodemographic and clinical characteristics were compared between the two groups using an independent $t$-test for normally distributed data, and the Mann-Whitney U test, chi-square test, or Fisher's exact test, for non-normally distributed data. Spearman correlation coefficients were calculated to explore the associations among psychiatric measures. To control for confounding effects, factors significantly associated with a previous history of suicide attempts in univariate analyses were adjusted for using 
Table 1. Baseline demographic and clinical characteristics with or without history previous suicide attempt

\begin{tabular}{|c|c|c|c|}
\hline \multirow{2}{*}{ Characteristic } & \multicolumn{2}{|c|}{ A previous history of suicide attempt } & \multirow{2}{*}{$p$ value } \\
\hline & Yes & No & \\
\hline Total & $20(23.0)$ & $67(77.0)$ & \\
\hline Sex, male & $11(55.0)$ & $40(59.7)$ & 0.708 \\
\hline Age $(y r)$ & $32.2 \pm 8.1$ & $35.1 \pm 10.1$ & 0.249 \\
\hline Educational level (yr) & $13.6 \pm 2.1$ & $14.5 \pm 4.2$ & 0.344 \\
\hline Duration of illness (mo) & $132.0(52.0-211.0)$ & $73.0(32.5-137.5)$ & 0.139 \\
\hline Alcohol drink, yes & $7(36.8)$ & $12(17.9)$ & 0.115 \\
\hline Smoking, yes & $4(21.1)$ & $21(31.3)$ & 0.383 \\
\hline Type of antipsychotics & & & 0.595 \\
\hline Risperidone & $7(35.0)$ & $12(17.9)$ & \\
\hline Paliperidone & $3(15.0)$ & $14(20.9)$ & \\
\hline Amisulpride & $4(20.0)$ & $14(20.9)$ & \\
\hline Aripiprazole & $3(15.0)$ & $9(13.4)$ & \\
\hline Olanzapine & $2(10.0)$ & $6(9.0)$ & \\
\hline Quetiapine & $1(5.0)$ & $5(7.5)$ & \\
\hline Others & $0(0.0)$ & $7(10.4)$ & \\
\hline Use of antidepressants, yes & $5(25.0)$ & $7(10.4)$ & 0.098 \\
\hline Use of anticonvulsants, yes & $6(30.0)$ & $12(17.9)$ & 0.344 \\
\hline \multicolumn{4}{|l|}{ PANSS } \\
\hline Positive & $15.9 \pm 5.2$ & $15.3 \pm 6.3$ & 0.697 \\
\hline Negative & $16.4 \pm 4.2$ & $17.4 \pm 4.9$ & 0.386 \\
\hline General & $34.2 \pm 10.5$ & $32.0 \pm 8.2$ & 0.323 \\
\hline Total & $66.4 \pm 18.1$ & $64.3 \pm 17.0$ & 0.640 \\
\hline BHS & $3(0-4)$ & $1(0-3)$ & 0.123 \\
\hline $\mathrm{BDI}$ & $14(4-21)$ & $6.5(1-14)$ & $0.036^{*}$ \\
\hline K-ISMI & $70(62-76)$ & $63(52-67)$ & $0.009^{*}$ \\
\hline SES & $22.8 \pm 4.9$ & $27.2 \pm 5.0$ & $0.001^{*}$ \\
\hline
\end{tabular}

Values are presented as number (\%), mean \pm standard deviation, or median (interquartile range).

*Statistically significant.

t-test, Mann-Whitney $U$ test, chi-square test, or Fisher's exact test, as appropriate.

PANSS, Positive and Negative Syndrome Scale; BDI, Beck Depression Inventory; BHS, Beck Hopelessness Scale; SES, Rosenberg Self-Esteem Scale; K-ISMI, Internalized Stigma of Mental IIIness scale.

analysis of covariance (ANCOVA). Variables that were not normally distributed were log-transformed for ANCOVA. Data were analyzed using the IBM SPSS Statistics for Windows software package (ver. 20.0; IBM Co., Armonk, NY, USA). A $p$-value of $<0.05$ was taken to indicate statistical significance (two-tailed).

\section{RESULTS}

A total of 87 subjects participated in the study. The sample included 51 males (58.6\%) and 36 females, with a mean age of $34.5 \pm 9.8$ years (range, $17-56$ years). Of these 87 participants, $20(23.0 \%)$ had attempted suicide. The results of group comparisons of sociodemographic and clinical characteristics, in accordance with the presence or absence of a previous suicide attempt, are presented in Table 1. There were no significant group differences according to suicide attempt history in sociodemographic or clinical factors, PANSS score, type of antipsychotic medication, use of antidepressants or mood stabilizer, and duration of illness. However, patients with a history of suicide at-
Table 2. Spearman correlations coefficients among clinical scales

\begin{tabular}{lllll}
\hline & \multicolumn{1}{c}{ SES } & K-ISMI & PANSS & BHS \\
\hline K-ISMI & $-0.588^{* \star *}$ & & & \\
PANSS & -0.092 & 0.138 & & \\
BHS & $-0.535^{* \star *}$ & $0.472^{* * *}$ & 0.033 & \\
BDI & $-0.560^{* \star *}$ & $0.521^{* * *}$ & $0.305^{\star *}$ & $0.478^{* *}$
\end{tabular}

${ }^{* *}$ Correlation is significant at the 0.01 level (2-tailed).

*** Correlation is significant at the 0.001 level (2-tailed)

K-ISMI, Internalized Stigma of Mental Illness scale; PANSS, Positive and Negative Syndrome Scale; BHS, Beck Hopelessness Scale, BDI: Beck Depression Inventory.

tempts exhibited significantly higher BDI $(p=0.036)$, K-ISMI $(p=0.009)$, and lower SES $(p=0.001)$ scores.

The correlation coefficients among psychiatric measures are presented in Table 2. SES scores were significantly negatively correlated with K-ISMI, BHS and BDI scores. K-ISMI scores were significantly positively correlated with BHS and BDI scores.

Variables (SES, K-ISMI and BDI scores) that were significantly associated with a previous history of suicide attempts were adjusted to investigate independent associa- 
tions between previous suicide attempts and these variables. ANCOVA revealed that self-esteem scores were significantly lower in patients with a history of previous suicide attempts than in those with no history, after controlling for K-ISMI and BDI scores (adjusted mean [standard error $]=24.4[0.9]$ vs. $26.7[0.5], \mathrm{F}=4.429, p=0.039$ ). However, scores on the BDI and K-ISMI did not significantly differ according to a previous history of suicide attempts after adjusting other variables $(p=0.982$ and 0.740 , respectively).

\section{DISCUSSION}

A previous history of suicide attempts is a strong risk factor for a future suicide attempt, and a strong predictor of completed suicide. ${ }^{26,27)}$ The Diagnostic and Statistical Manual of Mental Disorders, 5th edition (DSM-5) proposed 'Suicidal Behavior Disorder' as a new diagnosis in Section III (conditions requiring further study). The essential manifestation of suicidal behavior disorder is a suicide attempt. Defining suicidal behavior disorder as a separate diagnosis and identifying suicidal behavior in individual patients using medical records, are thought to be key steps within secondary and tertiary prevention strategies. ${ }^{28)}$ Therefore, our study to investigate associations between a previous history of suicide attempt and low self-esteem may have important and novel clinical implications with respect to the management of suicide risk in patients with schizophrenia.

In this study, a history of suicide attempts was associated with low self-esteem, depression, and high self-perceived stigma. In particular, low self-esteem was significantly associated with previous suicide attempts even after controlling for depression and self-perceived stigma. Therefore, when evaluating the suicide risk of patients with schizophrenia, clinicians should also assess their self-esteem. Furthermore, efforts should be made to improve self-esteem; this may be clinically important for the prevention of suicide attempts.

Low self-esteem was also associated with depression, hopelessness, and high perceived stigma, but not with degree of psychotic symptoms. Recognizing oneself as competent and worthy confers self-acceptance, self-respect and life satisfaction. High self-esteem may allow individuals to overcome difficulties and stressful life events, and protects against suicidal behaviors by decreasing vulnerability to depression. ${ }^{29)}$

Patients with low self-esteem may also have comorbid depression, which is a very well-known suicidal risk factor.
Low self-esteem and depression can independently increase the risk of suicide and may also act synergistically. ${ }^{30)}$ In this study, low self-esteem was significantly associated with a history of suicide attempt after controlling for depression. This suggests that, although low self-esteem is strongly associated with depression, it is not merely a subordinate construct. In particular, the relationship between suicidality and low self-esteem is very important regardless of the presence or absence of depression.

Patients with schizophrenia may have high levels of self-perceived stigma, which frequently prevents successful treatment and can lead to chronic social impairment. ${ }^{31)}$ Moreover, higher levels of self-perceived discrimination are associated with increased suicidal ideation and suicide attempts in patients with alcohol problems, and in patients with schizophrenia. ${ }^{14,32)}$ In this study, high levels of self-perceived stigma were significantly associated with a history of suicide attempts. High perceived stigma may induce low self-esteem and depression, which elevate suicide risk. Stigma affects individuals' trust of others, and causes feelings of worthlessness and lack of control; this can in turn lead to hopelessness and suicidal behavior. ${ }^{33)}$ Furthermore, suicide is associated with social isolation. ${ }^{34)}$ The interpersonal-psychological theory of suicidal behavior has three components: perceived burdensomeness, low belonging and social isolation, and an acquired ability for lethal self-injury; of these, social isolation has been demonstrated to be related to suicide. ${ }^{35)}$

In addition to suicide risk, self-esteem has several other clinical implications for patients with schizophrenia. First, low self-esteem is related to relapse. Previous study demonstrated that negative self-esteem was related to vulnerability to relapse; highly positive self-evaluation may delay relapse. ${ }^{36)}$ Second, patients with low self-esteem exhibit significantly higher rates of anxiety and affective comorbidity. ${ }^{15)}$ Third, low self-esteem affects quality of life. A previous study demonstrated that quality of life was significantly affected by various clinical characteristics, including satisfaction with self, general self-efficacy beliefs and depression. ${ }^{37)}$

As mentioned previously, low self-esteem is related to suicide in patients with schizophrenia for various reasons. Because it is modifiable, evaluating self-esteem, and employing interventions to address low self-esteem, is very important in patients with schizophrenia at high risk for suicide. In one previous study, cognitive behavioral therapy (CBT) reduced negative appraisals of loss arising from psychosis and improved self-esteem. ${ }^{38)}$ Therefore, CBT should be considered for the improvement of self-es- 
teem in patients with schizophrenia.

This study had a few limitations that should be acknowledged before generalizing the results. First, recall bias may have influenced the data on previous suicide attempts, because the assessment of previous suicide attempts depended on self-report (i.e., interview) measures. Second, we used a cross-sectional design such that causality between low self-esteem and a previous history of suicide attempts could not be assessed. Therefore, we cannot exclude the possibility that suicide attempts may decrease self-esteem. A longitudinal prospective study is required to ascertain whether low self-esteem at baseline predicts future suicide attempts.

In conclusion, this study suggests that low self-esteem is independently associated with suicidality in patients with schizophrenia. Low self-esteem appears to represent a psychological dimension that is closely related to suicide risk. Therefore, clinical attention should be paid to the evaluation and enhancement of low self-esteem in patients with schizophrenia and suicidality.

\section{Acknowledgments}

This study was supported by a grant of the Korean Health Technology R\&D, Ministry of Health \& Welfare, Republic of Korea (A120051).

\section{REFERENCES}

1. Nordentoft M, Laursen TM, Agerbo E, Qin P, Høyer EH, Mortensen PB. Change in suicide rates for patients with schizophrenia in Denmark, 1981-97: nested case-control study. BMJ 2004;329:261.

2. Fialko L, Freeman D, Bebbington PE, Kuipers E, Garety PA, Dunn G, et al. Understanding suicidal ideation in psychosis: findings from the Psychological Prevention of Relapse in Psychosis (PRP) trial. Acta Psychiatr Scand 2006; 114:177-186.

3. Kamali M, Kelly L, Gervin M, Browne S, Larkin C, O'Callaghan E. The prevalence of comorbid substance misuse and its influence on suicidal ideation among in-patients with schizophrenia. Acta Psychiatr Scand 2000;101:452-456.

4. Kim SW, Kim SJ, Mun JW, Bae KY, Kim JM, Kim SY, et al. Psychosocial factors contributing to suicidal ideation in hospitalized schizophrenia patients in Korea. Psychiatry Investig 2010;7:79-85.

5. Kontaxakis V, Havaki-Kontaxaki B, Margariti M, Stamouli S, Kollias C, Christodoulou G. Suicidal ideation in inpatients with acute schizophrenia. Can J Psychiatry 2004;49:476479.

6. Montross LP, Kasckow J, Golshan S, Solorzano E, Lehman D, Zisook S. Suicidal ideation and suicide attempts among middle-aged and older patients with schizophrenia spectrum disorders and concurrent subsyndromal depression. J Nerv Ment Dis 2008;196:884-890.

7. Tarrier N, Barrowclough C, Andrews B, Gregg L. Risk of non-fatal suicide ideation and behaviour in recent onset schizophrenia--the influence of clinical, social, self-esteem and demographic factors. Soc Psychiatry Psychiatr Epidemiol 2004;39:927-937.

8. Miles CP. Conditions predisposing to suicide: a review. J Nerv Ment Dis 1977; 164:231-246.

9. Caldwell CB, Gottesman II. Schizophrenics kill themselves too: a review of risk factors for suicide. Schizophr Bull 1990; 16:571-589.

10. Palmer BA, Pankratz VS, Bostwick JM. The lifetime risk of suicide in schizophrenia: a reexamination. Arch Gen Psychiatry 2005;62:247-253.

11. Inskip HM, Harris EC, Barraclough B. Lifetime risk of suicide for affective disorder, alcoholism and schizophrenia. Br J Psychiatry 1998;172:35-37.

12. Perenyi A, Forlano R. Suicide in schizophrenia. Neuropsychopharmacol Hung 2005;7:107-117.

13. Palmer CJ Jr. Suicide attempt history, self-esteem, and suicide risk in a sample of 116 depressed voluntary inpatients. Psychol Rep 2004;95:1092-1094.

14. Hor K, Taylor M. Suicide and schizophrenia: a systematic review of rates and risk factors. J Psychopharmacol 2010;24(4 Suppl):81-90.

15. Bhar S, Ghahramanlou-Holloway M, Brown G, Beck AT. Self-esteem and suicide ideation in psychiatric outpatients. Suicide Life Threat Behav 2008;38:511-516.

16. Petrie K, Brook R. Sense of coherence, self-esteem, depression and hopelessness as correlates of reattempting suicide. Br J Clin Psychol 1992;31:293-300.

17. Fulginiti A, Brekke JS. Escape from discrepancy: Self-esteem and quality of life as predictors of current suicidal ideation among individuals with schizophrenia. Community Ment Health J 2015;51:654-662.

18. American Psychiatric Association. Diagnostic and Statistical Manual of Mental Disorders. 4th ed. Washington, DC: American Psychiatric Association;1994.

19. Kay SR, Fiszbein A, Opler LA. The positive and negative syndrome scale (PANSS) for schizophrenia. Schizophr Bull 1987:13:261-276.

20. Yi JS, Ahn YM, Shin HK, An SK, Joo YH, Kim SH, et al. Reliability and validity of the Korean version of the Positive and Negative Syndrome Scale. J Korean Neuropsychiatr Assoc 2001;40:1090-1105.

21. Beck AT, Ward CH, Mendelson M, Mock J, Erbaugh J. An inventory for measuring depression. Arch Gen Psychiatry 1961; 4:561-571.

22. Hahn HM, Yum TH, Shin YW, Kim KH, Yoon DJ, Chung KJ. A standardization study of Beck Depression Inventory in Korea. J Korean Neuropsychiatr Assoc 1986;25:487-500.

23. Beck AT, Weissman A, Lester D, Trexler L. The measurement of pessimism: the hopelessness scale. J Consult Clin Psychol 1974;42:861-865.

24. Rosenberg M. Society and the adolescent self-image. NewJersy:Princeton University Press; 1965.

25. Hwang TY, Lee WK, Han ES, Kwon EJ. A study on the reliability and validity of the Korean version of internalized stigma of mental illness scale (K-ISMI). J Korean Neuropsychiatr Assoc 2006;45:418-426.

26. Suominen K, Isometsä E, Suokas J, Haukka J, Achte K, Lönnqvist J. Completed suicide after a suicide attempt: a 37-year follow-up study. Am J Psychiatry 2004;161:562563.

27. American Psychiatric Association. Diagnostic and Statistical Manual of Mental Disorders. 5th ed. Arlington, VA:American Psychiatric Publishing Company;2013.

28. Oquendo MA, Baca-Garcia E. Suicidal behavior disorder as 
a diagnostic entity in the DSM-5 classification system: advantages outweigh limitations. World Psychiatry 2014; 13:128-130.

29. Sharaf AY, Thompson EA, Walsh E. Protective effects of self-esteem and family support on suicide risk behaviors among at-risk adolescents. $J$ Child Adolesc Psychiatr Nurs 2009;22:160-168.

30. Kim SW, Stewart R, Kim JM, Shin IS, Yoon JS, Jung SW, et al. Relationship between a history of a suicide attempt and treatment outcomes in patients with depression. J Clin Psychopharmacol 2011;31:449-456.

31. Jackowska E. Stigma and discrimination towards people with schizophrenia--a survey of studies and psychological mechanisms. Psychiatr Pol 2009;43:655-670.

32. Perez-Rodriguez MM, Baca-Garcia E, Oquendo MA, Wang S, Wall MM, Liu SM, et al. Relationship between acculturation, discrimination, and suicidal ideation and attempts among US Hispanics in the National Epidemiologic Survey of Alcohol and Related Conditions. J Clin Psychiatry 2014;75:399-407.
33. Farrelly S, Jeffery D, Rüsch N, Williams P, Thornicroft G, Clement S. The link between mental health-related discrimination and suicidality: service user perspectives. Psychol Med 2015. [Epub ahead of print]

34. Durkheim É. Suicide: a study in sociology. New York:Glencoe Ill, Free Press; 1951.

35. Joiner T. Why people die by suicide. Cambridge, MA:Harvard University Press;2005.

36. Holding JC, Tarrier N, Gregg L, Barrowclough C. Self-esteem and relapse in schizophrenia: a 5-year follow-up study. $J$ Nerv Ment Dis 2013;201:653-658.

37. Chou CY, Ma MC, Yang TT. Determinants of subjective health-related quality of life (HRQoL) for patients with schizophrenia. Schizophr Res 2014;154:83-88.

38. Gumley A, Karatzias A, Power K, Reilly J, McNay L, O'Grady M. Early intervention for relapse in schizophrenia: impact of cognitive behavioural therapy on negative beliefs about psychosis and self-esteem. Br J Clin Psychol 2006; 45: 247-260. 